\title{
Remote Monitoring of Lead-Acid Battery Based on WLAN
}

\author{
Oka Danil Saputra ${ }^{1}$, Seog Chae ${ }^{2}$, Young Hyung Kim³ ${ }^{3}$, Soo Young Shin ${ }^{2 *}$ \\ ${ }^{1}$ Department of IT Convergence Engineering, \\ ${ }^{2}$ Department of Electronic Engineering, \\ ${ }^{3}$ Department of IT Convergence, \\ Kumoh National Institute of Technology, Gumi (730701), South Korea \\ *Corresponding Author: wdragon@kumoh.ac.kr
}

\begin{abstract}
Lead-acid battery is widely utilized to provide electricity in vehicle. There are dozens of system is used the lead-acid battery for power supply e.g., in electric car, mobile robot, electric forklift, etc. Nevertheless, overuse could significantly effect on life time of battery. In this paper, remote monitoring of lead-acid battery for electric forklift is proposed. We develop a data acquisition system to monitor battery parameters by developing dedicated hardware and software. Inter-integrated circuit (i2c), Analog to digital converter (ADC) and Universal Asynchronous Receiver/Transmitter (UART) are used as the protocol communication. The wireless local area network (WLAN) is used as the backbone networks. In experimental result, the data are successfully send from microcontroller to server. Furthermore, data is displayed on server monitor and stored in database storage.
\end{abstract}

Keywords: Remote Monitoring, Lead Acid, Wireless.

\section{Introduction}

In industrial environment, the utilization of forklift for transportation of goods is widely used. The main objective by using that vehicle to increases the productivity, efficiency and quality of work in factory. In the past, most of the manufacturing is used the forklift where the power supply from the fuel. Due to global warming issue and popularity of green technology, the new type of the forklift namely electric forklift is proposed to change the previous transports system $^{(1)}$. Thereafter, the clean energy battery system is offered from electric system. However, the system monitoring to check battery parameter such as remaining capacity, current, voltage, and state of charge are neglected by the authors. The problem such as overuse of battery could be another challenges to face. The effect regards those problem in viewpoint of technical is impact on reduce life time of battery, even in worst case lead to system failure.

This is attracted the researcher to overcome and find the solution by using remote monitoring. Elsewhere, the stationary lead-acid remote monitoring is proposed to obtain premature failure diagnose of lead-acid battery ${ }^{(2)}$. The other application of remote monitoring of lead-acid battery is proposed $\mathrm{in}^{(3)}$. The systems is applied to monitoring battery of base station. The main objective their scheme, provided and displayed data in dedicated website. So that, the user and technical team can access the battery status for troubleshooting and maintenance purpose. However, the system is designed only suitable for fixed battery. Meanwhile, the remote monitoring for electric vehicle is proposed in $^{(4)}$. To validate the proposed scheme, the software platform and dedicated hardware are developed. The aim is to monitoring battery status in real-time and automatically reports to supervision station and store data into database. In addition, the backbone technology for data transmission is used General Packet Radio Service (GPRS) ${ }^{(2-4)}$. However, the low data rate could be face in system since GPRS is assigned as the backbone networks and those technology unsuitable in relative small environment i.e., industry area.

To provide the remote monitoring system for electric forklift, in our prior work, we propose the system based on dedicated hardware and software ${ }^{(5-6)}$. Subsequently, a new scheme for remote monitoring of lead-acid battery is presented by considering the mobility of forklift. The initial work about lead-acid remote monitoring without consider the wireless is presented $\mathrm{in}^{(5)}$. Then, we extended the problem by including the wireless system as backbone $\mathrm{in}^{(6)}$. In our case, we focus to design remote battery monitoring for 
an electric forklift. The platform is designed to give real-time data for monitoring and to determine whether battery need to recharges or not. In this paper, the main objective of our scheme to provide creditable and valuable data. From viewpoint of utilities side, system is developed benefit to prevent battery damage caused by over using without notice low battery capacity. Eventually, the cost can be minimized and maintenance of battery is relatively easier than before. In summary, the technical contribution our paper are providing real-time battery monitoring, based on WLAN networks and considering the movement of forklift. From viewpoint of user, easy to maintenance and monitor the system. In term of economics side, the maintenance money is reduced. For other application, the system can be apply such as monitoring data in smart home system which mean system does not limited for battery monitoring only.

The rest of paper is organized as follows. The brief discussion of system model which consists of hardware, software and algorithm are presented in Section 2. Furthermore, in Section 3, test bed and experimental results of remote monitoring the battery parameters are depicted and explained, respectively. Last, the conclusion and future works is described in Section 4.

\section{System Model}

\subsection{Hardware}

The block diagram is presented for describe of our proposed system. The communication protocol for transfer data among device and microcontroller porting are depicted in Fig. 1. Firstly, the device measure parameter of battery which is installed. Bq34z110 module is used to measure the battery parameters such as remaining capacity, full charge capacity, voltage, state of charge and average current. The i2c protocol is applied to data transmission between device and microcontroller. Secondly, data of temperature and humidity around battery are obtained from chipcap-D sensor. ADC is utilized as protocol communication between device and microcontroller. Thirdly, after receiving data from sensor, the calculation and analysis part are implemented in microcontroller. Fourthly, the data is transferred to $\mathrm{Wi}-\mathrm{Fi}$ card (Wizfi) by using universal asynchronous receiver/transmitter (UART) protocol.

In our system, we divide into two part which are consisting microcontroller as transmitter and server as receiver where the communication between parts by using WLAN technology. The main advantage by using the

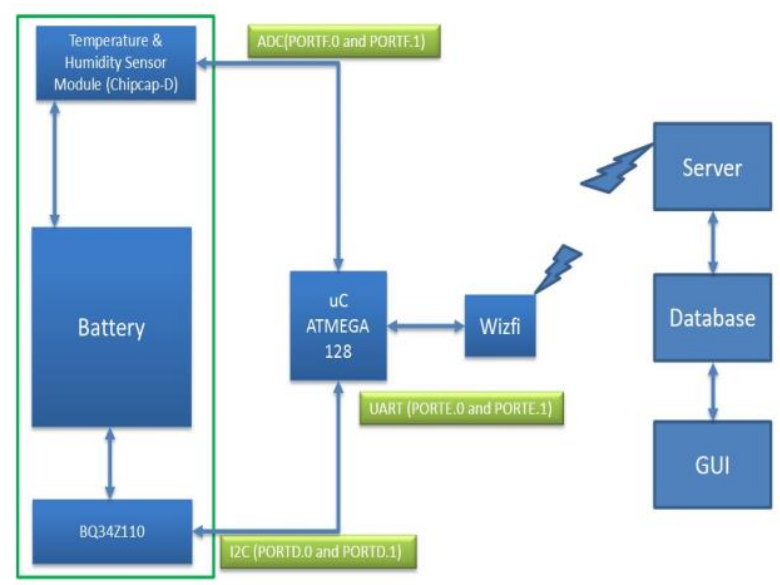

Fig. 1. Proposed system of remote monitoring of Battery

\begin{tabular}{|l|l|l|l|l|l|l|l|}
\hline Battery ID & cc & w & temp & rh & fc & SC & ac \\
\hline
\end{tabular}

Fig. 2. Data frame is used for communication between microcontroller and server

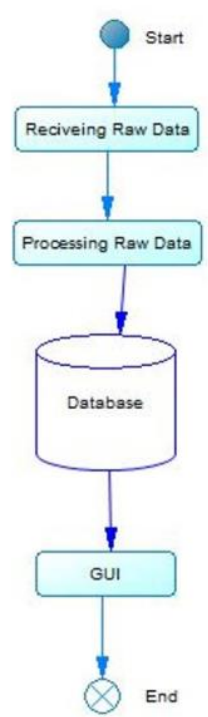

Fig. 3. Flowchart is used to received data in server

WLAN that mobility of forklift is independent to connect with any gateways and free wire is required.

\subsection{Software}

The software are classified into two part: microcontroller and server. Consequently, $\mathrm{C}$ language is used to code for processing the data in microcontroller. The data is collected into one data frame as shown in Fig. $2^{(5-6)}$. The objective is used the data frame to ensure only receiver with known data frame can decode the data. In beginning of frame 
the ID of battery should be define, since in our case n-number of battery is considered in networks. In details, the parameter assign as data which are consisting remaining capacity (cc), voltage (vv), temperature (temp), humidity (rh), full charge capacity (fc), state of charge (sc) and average current (ac). The data is gathered into one data frame and sent periodically from Wizfi to server by using WLAN as the transfer bridge. In server side, the data is received by following flowchart in Fig. 3. Java language is used to design the socket programming. In receiver side, data is divided into column by following the data frame. Next part, data is stored in SQL database. Finally, the data is presented GUI. The main function of GUI is observed the data and at same time easy to monitor the battery parameters.

\section{Result}

In this section, the experimental result is obtained and presented. The lead-acid battery and device that are implemented for test bed shown in Fig. 4. The scenario of testing process are started from read data by using the sensors device and collected to microcontroller. To validate the data is matched according the actual data, the direct wire data transfer from microcontroller to terminal by using UART protocol is evaluated and the result shown in Fig. 5.

Furthermore, the IP of wizfi is assigned before the transmitted the data. In our case, 192.168.0.117:4000 is predefined address and similarly, the IP shown database is matched with the predefine IP. After the connection is established, data transmission is running communication in system. As shown in the result of Fig. 6, the data is received periodically form the source with interval period 1 minutes. As shown, the different data are presented in Fig. 5. and Fig. 6 . The reason behind the results because different testing time so that the data generated is not similar.

The battery parameters results in database is displayed in GUI while data are provided in table format. In Fig. 7, there are several feature design for improve GUI functionality. The connect button is used to made the connection between GUI and database while the clear and show button are used for delete and shown data, respectively. Our GUI also accommodate to export data from GUI into excel file. This function is used to give the user to further analysis healthy level of battery. Last, for clarify the validity the data, we compared the received data GUI and data from microcontroller. In summary, all the data is successfully transmitted and received.

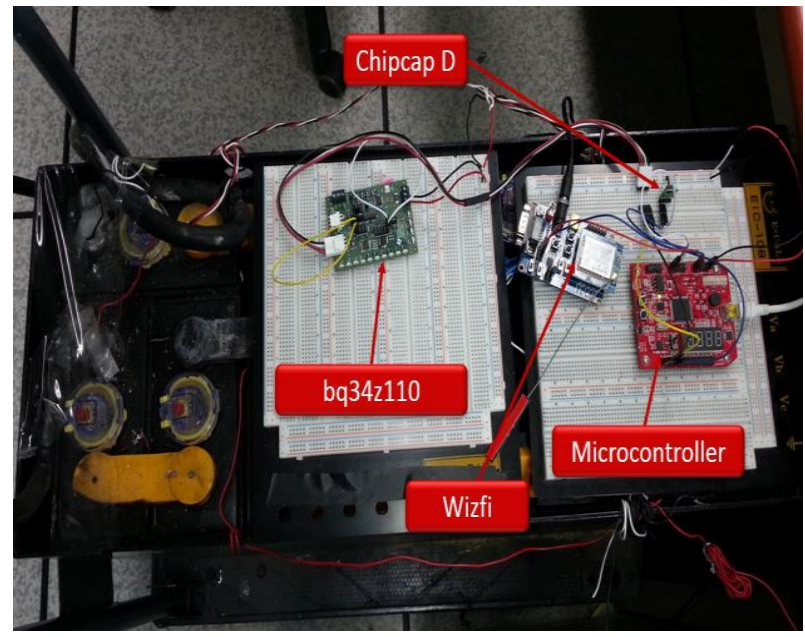

Fig. 4. Forklift battery test bed.

Serial port COM6 opened
00012926001200160039399000770000
00012926001200200041399000770000
00012926001200200041399000770000
00012926001200200041399000770000
00012926001200160039399000770000
00012926001200160039399000770000
Serial port COM6 closed

Fig. 5. UART data test by using serial terminal port.

\section{Conclusions and Future Works}

In this paper, remote monitoring of lead-acid battery is proposed. The examination and evaluation of remote monitoring battery as our main objective. The target of the system is provide the valuable and real-time data from electric forklift power supply. The WLAN networks is assigned as the core network to transmission the data between device and server. In experimental result shown, the system successfully sent data from batteries to server. For future works, we will discover to present the data into android GUI. We desire to implement our platform in real industry environment with consider $n$-number of battery. In that scenario, we will manage how to collect data from several battery in real-time.

\section{Acknowledgment}

This research was supported by the MSIP (Ministry of Science, ICT and Future Planning), Korea, under the Global IT Talent support program (NIPA-2014-H0904-14-1005) supervised by the NIPA (National IT Industry Promotion Agency). 


\begin{tabular}{|c|c|c|c|c|c|c|c|c|c|c|c|}
\hline$\nabla$ & $\begin{array}{c}\text { Number } \\
\nabla\end{array}$ & r Battery_ID & $\mathbb{P}$ & Port & Remaining_Capacity & Voltage & Temperature & Humidity & Full_Charge_Capacity & State_Of_Charge & Average_Current \\
\hline zit Salin O Hapus & 13 & 30001 & 192.168.0.117 & 4000 & 2926 & 0012 & 0020 & 0041 & 3990 & 0074 & 0000 \\
\hline git Salin $\odot$ Hapus & 12 & 20001 & 192.168.0.117 & 4000 & 2926 & 0012 & 0020 & 0041 & 3990 & 0074 & 0000 \\
\hline Zit Salin $\Theta$ Hapus & 11 & 10001 & 192.168.0.117 & 4000 & 2926 & 0012 & 0020 & 0041 & 3990 & 0074 & 0000 \\
\hline jat Salin $\bigcirc$ Hapus & 10 & 00001 & 192.168.0.117 & 4000 & 2926 & 0012 & 0020 & 0041 & 3990 & 0074 & 0000 \\
\hline gat: Salin (- Hapus & s & 90001 & 192.168.0.117 & 4000 & 2926 & 0012 & 0020 & 0041 & 3990 & 0074 & 0000 \\
\hline jut Salin 0 Hapus & s & 80001 & 192.168.0.117 & 4000 & 2926 & 0012 & 0020 & 0041 & 3990 & 0074 & 0000 \\
\hline
\end{tabular}

Pilih Semua Dengan pilihan: Ubah O Hapus Ekspor

Fig. 6. Data are stored in server database.

\begin{tabular}{|c|c|c|c|c|c|c|c|c|c|}
\hline \multicolumn{3}{|l|}{ 驺 } & \multicolumn{4}{|c|}{ FORKLIFT BATTERY REPORTER } & & & $-\square x$ \\
\hline \multicolumn{10}{|c|}{ FORKLIFT BATTERY REPORTER } \\
\hline Connect & \multicolumn{2}{|c|}{ Clear } & & & & & & & \\
\hline Battery_ID & IP & Port & Remaining_C... & Voltage & Temperature & Humidity & Full_Charge_... & State_Of_Cha... & Average_Curr... \\
\hline 0001 & 192.168.0.117 & 4000 & 2926 & 0012 & 0020 & 0041 & 3990 & 0074 & 0000 \\
\hline 0001 & 192.168.0.117 & 4000 & 2926 & 0012 & 0020 & 0041 & 3990 & 0074 & 0000 \\
\hline 0001 & 192.168.0.117 & 4000 & 2926 & 0012 & 0020 & 0041 & 3990 & 0074 & 0000 \\
\hline 0001 & 192.168.0.117 & 4000 & 2926 & 0012 & 0020 & 0041 & 3990 & 0074 & 0000 \\
\hline 0001 & 192.168.0.117 & 4000 & 2926 & 0012 & 0020 & 0041 & 3990 & 0074 & 0000 \\
\hline \multicolumn{2}{|c|}{ Show } & & & & & & Print & & Close \\
\hline
\end{tabular}

Fig. 7. Data are displayed in GUI.

\section{References}

(1) T.M. Keränen, H. Karimäki, J. Viitakangas, J. Vallet, J. Ihonen, P. Hyötylä, H. Uusalo, T. Tingelöf,: "Development of integrated fuel cell hybrid power source for electric forklift", Journal of Power Source, Vol. 196, pp. 9058-9068, 2011

(2) Rosolem, M.F.N.C., Rangel Pessenti, G., Beck, R.F., Ribeiro dos Santos, G., Torquato Arioli, V., Lopes, P.H.O., : "Stationary lead-acid batteries remote monitoring system", in Proceeding Telecommunication Energy Conference, pp. 1-7, 2011

(3) Xia Zezhong, Su Hongliang, Liu Ting, : "Remote Monitoring System of Lead-Acid Battery Group Based on GPRS", in Proceeding Electrical and Control Engineering (ICECE), pp. 4023-4026, 2010

(4) Min Luo, Yong Xiao, Wei-Ming Sun, Zhiping Wang, : "Online Battery Monitoring System Based on GPRS for Electric Vehicles", in Proceeding Intelligent HumanMachine Systems and Cybernetics, pp. 122-125, 2013

(5) Saputra O. D., Shin S. Y., : "Implementation of Lead Acid Battery Remote Monitoring for Electric Forklift in Industrial Environment", in Proceeding International Workshop on Industrial IT Convergence, pp. 66-68, 2014

(6) Saputra O. D., Shin S. Y., : "Network-based Wireless for Remote Monitoring Lead-Acid Battery", appear in Proceeding Fall KICS Conference, 2014 